\title{
CULTURA DE SEGURANÇA DO PACIENTE EM TRÊS INSTITUIÇÕES HOSPITALARES: PERSPECTIVA DA EQUIPE DE ENFERMAGEM
}

\author{
PATIENT SAFETY CULTURE IN \\ THREE HOSPITAL INSTITUTIONS: \\ NURSING TEAM PERSPECTIVE
}

\section{CULTURA DE SEGURIDAD DEL PACIENTE EN TRES INSTITUCIONES HOSPITALARIAS: PERSPECTIVA DEL EQUIPO DE ENFERMERÍA}

\author{
Graziele de Carvalho Lemos ${ }^{1}$ \\ Luciana Regina Ferreira da Mata ${ }^{2}$ \\ Helen Cristiny Teodoro Couto Ribeiro ${ }^{3}$ \\ Aline Carrilho Menezes ${ }^{4}$ \\ Carolina de Sousa Penha ${ }^{5}$ \\ Ronise Malaquias Carlos Valadares ${ }^{6}$ \\ Marlene Santos Rios Castro ${ }^{7}$
}

\begin{abstract}
Como citar este artigo: Lemos GC, Mata LRF, Ribeiro HCTC, Menezes AC, Penha CS, Valadares RMC, et al. Cultura de segurança do paciente em três instituições hospitalares: perspectiva da equipe de enfermagem. Rev baiana enferm. 2022;36:e43393.

Objetivo: avaliar a cultura de segurança do paciente em três instituições hospitalares, sob a perspectiva da equipe de enfermagem. Método: estudo quantitativo e transversal analítico realizado com 303 profissionais de enfermagem de três hospitais mineiros. Aplicou-se o questionário Hospital Survey on Patient Safety Culture. Foram realizadas análises estatísticas descritivas e inferencial bivariada. Resultados: pela perspectiva da enfermagem, nenhum dos três hospitais apresentou dimensões fortes para a cultura de segurança. O hospital filantrópico obteve melhores avaliações em quatro dimensões em relação aos hospitais da rede pública. A dimensão com maior fragilidade em todos os hospitais foi "Retorno da informação e comunicação sobre erro". Conclusão: sob a perspectiva das equipes de enfermagem, todas as dimensões da cultura de segurança do paciente revelaram fragilidades nos hospitais em estudo.
\end{abstract}

Descritores: Segurança do Paciente. Equipe de Enfermagem. Qualidade da Assistência à Saúde. Cultura Organizacional.

Enfermeira. Mestre em Ciências. Enfermeira do Núcleo de Segurança do Paciente do Complexo de Saúde São João de Deus. Divinópolis, Minas Gerais, Brasil. https://orcid.org/0000-000 I-6356-554l.

Enfermeira. Doutora em Enfermagem. Professora Associada da Universidade Federal de Minas Gerais. Belo Horizonte, Minas Gerais, Brasil. lucianarfmata@gmail.com. https://orcid.org/0000-0002-5080-4643.

3 Enfermeira. Doutora em Enfermagem. Professora Adjunta da Universidade Federal São João del-Rei. Divinópolis, Minas Gerais, Brasil. https://orcid.org/0000-000 I9365-7228.

4 Enfermeira. Mestre em Ciências. Professora da Escola Técnica do Complexo de Saúde São João de Deus. Divinópolis, Minas Gerais, Brasil. https://orcid.org/0000$0001-7658-4039$.

Médica. Residente em Dermatologia no Hospital da Polícia Militar de Minas Gerais. Belo Horizonte, Minas Gerais, Brasil. https://orcid.org/0000-0003-I I l 0-8095

Enfermeira. Mestre em Ciências. Assessora de Gestão Estratégica e Qualidade do Hospital Júlia Kubitschek. Belo Horizonte, Minas Gerais, Brasil. https://orcid.org/0000-0002-9318-4835.

Enfermeira. Especialista em auditoria e segurança do paciente. Enfermeira PENF IV B do Hospital Eduardo de Menezes. Belo Horizonte, Minas Gerais, Brasil. https://orcid.org/0000-000 I-9560-4517. 
Objective: to evaluate the patient safety culture in three hospital institutions, from the perspective of the nursing team. Method: quantitative and cross-sectional analytical study conducted with 303 nursing professionals from three hospitals in Minas Gerais. The Hospital Survey on Patient Safety Culture questionnaire was applied. Descriptive and inferential bivariate statistical analyses were performed. Results: from the perspective of nursing, none of the three hospitals presented strong dimensions for the safety culture. The philanthropic hospital obtained better evaluations in four dimensions in relation to public hospitals. The dimension with greater fragility in all hospitals was "Return of information and communication about error". Conclusion: from the perspective of nursing teams, all dimensions of the patient safety culture revealed weaknesses in the hospitals under study.

Descriptors: Patient Safety. Nursing, Team. Quality of Health Care. Organizational Culture.

Objetivo: evaluar la cultura de seguridad del paciente en tres instituciones hospitalarias, desde la perspectiva del equipo de enfermería. Método: estudio analítico cuantitativo y transversal realizado con 303 profesionales de enfermería de tres hospitales de Minas Gerais. Se aplicó el cuestionario Hospital Survey on Patient Safety Culture. Se realizaron análisis estadísticos bivariados descriptivos e inferenciales. Resultados: desde la perspectiva de la enfermería, ninguno de los tres hospitales presentó dimensiones fuertes para la cultura de seguridad. El hospital filantrópico obtuvo mejores evaluaciones en cuatro dimensiones en relación a los hospitales públicos. La dimensión con mayor fragilidad en todos los hospitales fue "Retorno de información y comunicación sobre error". Conclusión: desde la perspectiva de los equipos de enfermería, todas las dimensiones de la cultura de seguridad del paciente revelaron debilidades en los hospitales en estudio.

Descriptores: Seguridad del Paciente. Grupo de Enfermería. Calidad de la Atención de Salud. Cultura Organizacional.

\section{Introdução}

A segurança do paciente é definida como uma estrutura de atividades devidamente organizadas, responsáveis por criar processos, comportamentos e ambientes na área da saúde que reduzem os riscos e danos evitáveis, além de amenizar o impacto da sua ocorrência ${ }^{(1)}$. A assistência insegura impacta de maneira negativa na organização hospitalar e no cuidado ao paciente, pois pode aumentar o tempo de internação, intensificar a ocorrência de eventos adversos e de danos transitórios ou permanentes ao paciente e, principalmente, elevar os custos assistenciais ${ }^{(2)}$.

Sendo assim, é essencial o fortalecimento da cultura de segurança do paciente nas organizações de saúde como forma de melhoria contínua na prestação do cuidado e na estratégia efetiva, o que permite o gerenciamento dos riscos com foco na mitigação de erros. Existem diversos fatores a serem desenvolvidos para o fortalecimento da cultura de segurança nas organizações de saúde. A comunicação entre a equipe e o paciente é um desses fatores relevantes, uma vez que permite estabelecer vínculo de confiança, o que melhora a percepção dos profissionais no que tange às medidas preventivas em prol de uma assistência mais qualificada e segura ${ }^{(3)}$.
A cultura de segurança é um padrão integrado de comportamento individual e coletivo, baseado em crenças, percepções, atitudes, valores e padrões de comportamento compartilhados ${ }^{(4)}$. Pode determinar o estilo, a proficiência, o compromisso da administração e a gestão da organização de saúde em relação à segurança do paciente, substituindo a cultura punitiva pelo aprendizado baseado em erros ${ }^{(4)}$. Uma cultura de segurança forte incentiva o trabalho em equipe, a notificação de eventos adversos, a liberdade de expressão, a transparência e o aprendizado pautado nos erros, além de envolver a gestão, os profissionais e o paciente na melhoria do cuidado ${ }^{(5)}$.

A enfermagem é considerada uma profissão indispensável para identificação, relato e mensuração de erros na assistência à saúde ${ }^{(6)}$, visto que é responsável por grande parte das ações assistenciais nos hospitais. Portanto, é importante avaliar a percepção da equipe de enfermagem quanto à cultura de segurança do paciente em instituições hospitalares, para, ao extrair informações sobre essas, obter um diagnóstico situacional que possibilite o estabelecimento de estratégias de melhoria e fortalecimento da assistência ${ }^{(7)}$. 
Em meados dos anos 2000, dentre as estratégias de gestão desenvolvidas, evidenciou-se a elaboração de instrumentos e questionários autoaplicáveis, voltados para avaliação e compreensão da cultura de segurança nas organizações de saúde e o seu reflexo na qualidade da assistência prestada ao paciente. Tratam-se de instrumentos alicerçados nas dimensões prioritárias da segurança do paciente, que possuem vantagens metodológicas de baixo custo para as instituições e garantem ainda a confiabilidade e o sigilo das informações fornecidas pelos profissionais entrevistados ${ }^{(8)}$.

A avaliação da cultura de segurança nas organizações hospitalares é uma ferramenta útil para gestores e líderes em saúde, incluindo a liderança em enfermagem. Esta avaliação pode ser obtida por meio da percepção dos profissionais de saúde, de forma a apontar as áreas que necessitam de melhorias, direcionando intervenções e delineamento de estratégias para mitigação e prevenção das falhas e erros assistenciais ${ }^{(6)}$.

Para tal finalidade, destaca-se o instrumento Hospital Survey on Patient Safety Culture (HSOPSC) $^{(4)}$, por apresentar uma abordagem dimensional em escalas de múltiplos itens. Tem sido amplamente utilizado para avaliar a cultura de segurança do paciente nas instituições hospitalares de países desenvolvidos e em desenvolvimento, com vistas à identificação das áreas que necessitam de direcionamento das ações preventivas de segurança do paciente. Foi desenvolvido pela Agency for Healthcare Research and Quality (AHRQ), nos Estados Unidos da América. O HSOPSC apresenta boas propriedades psicométricas ${ }^{(8)}$, já foi utilizado em mais de 60 países, adaptado e validado em mais de 30 culturas $^{(9)}$. Salienta-se que esse instrumento ${ }^{(4)}$ foi adaptado culturalmente em $2013^{(10)}$ e validado em 2016 para o contexto brasileiro. Apresentou coeficiente alfa de Cronbach de 0,91, o que lhe confere boa confiabilidade $\mathrm{e}^{(11)}$.

Nesse sentido, justificam-se os estudos na área para melhoria da prática dos profissionais da enfermagem e da gestão das organizações de saúde hospitalares no planejamento das ações direcionadas à cultura de segurança do paciente.
Avaliar a cultura de segurança em um serviço é uma estratégia fundamental para o apontamento de não conformidades e fragilidades das áreas assistenciais e administrativas, o que possibilita o direcionamento das intervenções para mitigação e prevenção de erros na assistência.

Nessa perspectiva, o objetivo deste estudo foi avaliar a cultura de segurança do paciente em três instituições hospitalares, sob a perspectiva da equipe de enfermagem.

\section{Método}

Estudo de abordagem quantitativa, do tipo transversal analítico, conforme as diretrizes para estudos observacionais, Strengthening the Reporting of Observational Studies in Epidemiology (STROBE) ${ }^{(12)}$. A pesquisa foi realizada em três instituições hospitalares do estado de Minas Gerais, que se diferem quanto ao perfil de atendimento, filosofia e política institucionais. Neste estudo, identificou-se os hospitais por meio de letras alfabéticas A, B e C.

O hospital A é filantrópico, conveniado ao Sistema Único de Saúde (SUS) e a outros convênios particulares. Desenvolve atividades de ensino, assistência geral, ambulatorial e de alta complexidade. Dispõe de 372 leitos e possui 540 profissionais de enfermagem, sendo 158 auxiliares, 220 técnicos de enfermagem e 162 enfermeiros. O Núcleo de Segurança do Paciente (NSP) foi implantado em 2016. O hospital B é conveniado ao SUS e credenciado como hospital de ensino. Possui gestão estadual e contempla serviços de média a alta complexidade, sendo referência para doenças respiratórias complexas. Dispõe de 369 leitos e possui 754 profissionais de enfermagem, sendo 136 auxiliares de enfermagem, 476 técnicos de enfermagem e 142 enfermeiros. O NSP foi implementado no ano de 2015. O hospital C é conveniado ao SUS e é também credenciado como hospital de ensino. Possui gestão estadual, contempla serviços de média complexidade com ênfase nas doenças infectocontagiosas e na dermatologia sanitária. Dispõe de 110 leitos e possui 246 profissionais de enfermagem: 22 auxiliares de enfermagem, 
178 técnicos de enfermagem e 46 enfermeiros. O NSP foi criado no ano de 2016.

A população do estudo foi composta por 1.540 profissionais de enfermagem (auxiliares, técnicos de enfermagem e enfermeiros). Para definição do tamanho amostral, foram considerados como parâmetros o nível de significância 5\% e a margem de erro de $5 \%$ alfa ou erro tipo I ${ }^{(13-14)}$, o que resultou em uma amostra de 303 participantes. Utilizou-se a técnica de amostragem estratificada proporcional, a fim de manter a representatividade da população, por se tratar de três hospitais com características diferentes e de três categorias profissionais. Para isso, utilizou-se o método de estimação de proporções para populações finitas com alocação proporcional por categoria profissional (auxiliares, técnicos de enfermagem e enfermeiros) e por hospital (A, $\mathrm{B}$ e C). Portanto, a amostra que inferiu representatividade foi composta de 106 profissionais de enfermagem no hospital A, 148 no hospital B e 49 no hospital C. Assim, no hospital A participaram 32 auxiliares de enfermagem, 42 técnicos de enfermagem e 32 enfermeiros; no hospital B, 27 auxiliares de enfermagem, 92 técnicos de enfermagem e 29 enfermeiros; no hospital C, 5 auxiliares de enfermagem, 35 técnicos de enfermagem e 9 enfermeiros.

Os critérios de inclusão foram estabelecidos conforme orientações dos autores do HSOPSC ${ }^{(4)}$ : carga horária igual ou superior a 20 horas semanais e atuação profissional mínima de 6 meses no hospital. Foram excluídos os profissionais de enfermagem que estavam de férias, licença-saúde ou outra forma de afastamento durante o período da coleta de dados.

Para a coleta de dados, cada hospital forneceu uma lista dos profissionais de enfermagem com a função e o turno de trabalho. Os nomes dessa lista foram inseridos em uma planilha construída no software Microsoft Excel ${ }^{\circledR}$. Posteriormente, realizou-se o sorteio dos participantes de cada estrato, por meio de um site de domínio público gratuito. A coleta de dados ocorreu no período de janeiro a setembro de 2017.

A abordagem dos profissionais de enfermagem pelos pesquisadores foi realizada em seu local de trabalho, de forma individualizada. Após a concordância em participar, foi-lhes entregue um envelope opaco não identificado, contendo os instrumentos de coleta e as orientações de preenchimento. O recolhimento dos envelopes ocorreu 7 dias após a entrega, por meio de uma urna colocada no posto de enfermagem do setor. Durante a coleta de dados, 9 profissionais de enfermagem recusaram-se a participar da pesquisa (2 profissionais do hospital A, 5 do hospital B e 2 do hospital C). Após 4 tentativas de recolhimento dos questionários preenchidos, 12 profissionais desistiram de participar (2 do hospital A e 10 do hospital B). Conforme as orientações da $\mathrm{AHRQ}^{(4)}, 3$ instrumentos foram excluídos (2 referentes ao hospital A e 1 ao hospital B) por estarem preenchidos de forma incompleta e de maneira sequenciada, isto é, coincidindo todas as respostas do instrumento. Os participantes desistentes ou que se recusaram a participar da pesquisa foram substituídos por outros profissionais da mesma categoria, mediante um novo sorteio pela planilha elaborada.

A versão brasileira do questionário HSOPSC $^{(10-11)}$, disponibilizada desde 2004 pela AHRQ, foi utilizada após a autorização dos autores. O HSOPSC é um questionário autoaplicável, composto por 9 seções, 42 itens organizados em 12 dimensões: 1 - Trabalho em equipe dentro das unidades ( 4 itens); 2 - Expectativas sobre o seu supervisor/chefe e ações promotoras da segurança do paciente (4 itens); 3 - Aprendizado organizacional-melhoria contínua (3 itens); 4 - Apoio da gestão para segurança do paciente (3 itens); 5 - Percepção geral da segurança do paciente ( 4 itens); 6 - Retorno da informação e comunicação sobre erro (3 itens); 7 - Abertura na comunicação (3 itens); 8 - Frequência de relato de eventos ( 3 itens); 9 - Trabalho em equipe entre as unidades (4 itens); 10 - Adequação de profissionais (4 itens); 11- Passagem de plantão ou de turno/transferências ( 4 itens); e 12 - Respostas não punitivas aos erros (3 itens).

O HSOPSC compõe-se de uma escala do tipo Likert de cinco pontos relativos à concordância e à ocorrência: 1 - Discordo totalmente; 2 - Discordo; 3 - Não concordo nem discordo; 
4 - Concordo; 5 - Concordo totalmente; e 1 - Nunca; 2 - Raramente; 3 - Às vezes; 4 - Quase sempre; e 5 - Sempre. Para garantir respostas consistentes, 18 questões são reversas, isto é, quando o participante discorda do item formulado negativamente e a sua opinião é expressa de forma positiva. Além disso, contempla duas questões que são avaliadas separadamente: nota de segurança do paciente (0-10) e número de eventos adversos notificados nos últimos 12 meses ${ }^{(4,10-11)}$. Além disso, foi aplicado um questionário de caracterização do perfil sociodemográfico e profissional dos participantes com as seguintes variáveis: sexo, data de nascimento, situação conjugal, categoria profissional, nível de escolaridade, data de admissão, cargo/função, turno de trabalho, outros vínculos empregatícios, tempo de trabalho no hospital e carga-horária total de trabalho no hospital.

Para análise dos dados utilizou-se o software Statistical Package for the Social Sciences ${ }^{\circledR}$ (SPSS) versão 21.0. Realizou-se a análise descritiva das variáveis e a apresentação dos dados por meio de tabelas de distribuição de frequências, medidas de tendência central (média) e variabilidade (desvio-padrão). Para avaliar a cultura de segurança do paciente considera-se a soma dos resultados das 12 dimensões e multiplica-se por 100. Já para a análise de cada dimensão do instrumento HSOPSC, os percentuais de respostas positivas referentes às dimensões da escala foram calculados por meio da média do percentual de respostas positivas estimado para cada hospital, utilizando-se a fórmula ${ }^{(4)}$ :

\% de respostas positivas da dimensão X do HSOPSC $=$ número de respostas positivas aos itens da dimensão $\mathrm{X} \div$ número total de respostas válidas aos itens da dimensão $\mathrm{X} \times 100$

Dessa forma, a porcentagem de respostas positivas de cada dimensão do HSOPSC é o resultado do número de respostas positivas aos itens da dimensão X dividido pelo número total de respostas válidas (positivas, neutras e negativas, excluindo-se os dados ausentes) aos itens da dimensão X multiplicado por 100.

São consideradas respostas positivas, as opções: concordo fortemente, concordo, sempre e frequentemente; as opções para as respostas negativas são: discordo totalmente, discordo, nunca e raramente; para respostas neutras, são opções: não concordo e nem discordo ${ }^{(4)}$. Conforme a $\mathrm{AHRQ}^{(4,9)}$, as áreas consideradas fortes para a cultura de segurança possuem 75,0\% das respostas positivas, áreas neutras $<75,0 \%$ a $>50,0 \%$ das respostas positivas, e áreas fragilizadas ou críticas, $\leq 50,0 \%$ das respostas positivas.

O teste Kolmogorov-Smirnov foi utilizado para testar a normalidade das variáveis numéricas. Para a comparação de três ou mais grupos foi utilizado o teste não-paramétrico Kruskall-Wallis. Para a comparação dos escores das dimensões do instrumento HSOPSC por grupos dois a dois, foi utilizado o teste não-paramétrico Mann-Whitney, com correção de Bonferroni (valor-p significativo menor que 0,05 dividido pelo número de comparações). A comparação dos dados de natureza categórica foi realizada com base nos testes Qui-quadrado de Pearson e exato de Fisher. O nível de significância adotado foi de 5\%.

Os participantes assinaram o Termo de Consentimento Livre Esclarecido (TCLE) de acordo com a Resolução n. 466/2012, do Conselho Nacional de Saúde. O estudo obteve aprovação do Comitê de Ética em Pesquisa (CEP) da Universidade Federal São João del-Rei e instituições coparticipantes sob Parecer n. 1.785.549, Certificado de Apresentação de Apreciação Ética (CAAE) no 60925516.6.0000.5545.

\section{Resultados}

A equipe de enfermagem que participou do estudo, em sua maioria, era do sexo feminino, 252 (86,3\%), sendo 84 (80,0\%) do hospital A, 130 $(93,5 \%)$ do hospital B e $38(79,2 \%)$ do hospital C. A média de idade foi de $35,7( \pm 10,4), 43,7( \pm 10,0)$ e 40,8 $( \pm 7,4)$ respectivamente (Tabela 1$)$. 
Tabela 1 - Caracterização da equipe de enfermagem nas três instituições hospitalares. Divinópolis, Minas Gerais, Brasil - 2018. (N=303)

\begin{tabular}{|c|c|c|c|c|}
\hline $\begin{array}{l}\text { Caracteristicas da equipe de } \\
\text { enfermagem }\end{array}$ & Hospital A & Hospital B & Hospital C & Valor-p (1) \\
\hline \multicolumn{5}{|l|}{ Categoria profissional $(n=291)$} \\
\hline Enfermeiro & $31(31,0 \%)$ & $32(22,5 \%)$ & $9(18,4 \%)$ & \multirow[t]{3}{*}{$0,013(2)$} \\
\hline Técnico de Enfermagem & $51(51,0 \%)$ & $95(66,9 \%)$ & $38(77,6 \%)$ & \\
\hline Auxiliar de Enfermagem & $18(18,0 \%)$ & $15(10,6 \%)$ & $2(4,1 \%)$ & \\
\hline \multicolumn{5}{|l|}{ Turno de trabalho $(n=274)$} \\
\hline Diurno & $56(60,9 \%)$ & $108(80,6 \%)$ & $35(72,9 \%)$ & \multirow[t]{3}{*}{$0,021(2)$} \\
\hline Noturno & $31(33,7 \%)$ & $23(17,2 \%)$ & $12(25,0 \%)$ & \\
\hline Rodízio & $5(5,4 \%)$ & $3(2,2 \%)$ & $1(2,1 \%)$ & \\
\hline \multicolumn{5}{|l|}{ Presta assistência ao paciente } \\
\hline Não & $8(7,6 \%)$ & $18(12,1 \%)$ & $8(16,3 \%)$ & \multirow[t]{2}{*}{0,252} \\
\hline $\operatorname{Sim}$ & $97(92,4 \%)$ & $131(87,9 \%)$ & $41(83,7 \%)$ & \\
\hline \multicolumn{5}{|c|}{$\begin{array}{l}\text { Executa liderança na instituição } \\
(n=265)\end{array}$} \\
\hline Não & $70(77,8 \%)$ & $104(81,9 \%)$ & $44(91,7 \%)$ & \multirow[t]{2}{*}{0,125} \\
\hline $\operatorname{Sim}$ & $20(22,2 \%)$ & $23(18,1 \%)$ & $4(8,3 \%)$ & \\
\hline \multicolumn{5}{|c|}{ Possui vínculo empregatício $(n=273)$} \\
\hline Não & $68(74,7 \%)$ & $93(69,4 \%)$ & $33(68,8 \%)$ & \multirow[t]{2}{*}{0,638} \\
\hline $\operatorname{Sim}$ & $23(25,3 \%)$ & $41(30,6 \%)$ & $15(31,3 \%)$ & \\
\hline \multicolumn{5}{|c|}{ Tempo de trabalho no hospital $(n=302)$} \\
\hline 1 a 5 anos & $44(41,9 \%)$ & $68(45,9 \%)$ & $25(51,0 \%)$ & \multirow[t]{5}{*}{0,624} \\
\hline 6 a 10 anos & $30(28,6 \%)$ & $34(23,0 \%)$ & $13(26,5 \%)$ & \\
\hline 11 a 15 anos & $11(10,5 \%)$ & $13(8,8 \%)$ & $4(8,2 \%)$ & \\
\hline 16 a 20 anos & $11(10,5 \%)$ & $12(8,1 \%)$ & $5(10,2 \%)$ & \\
\hline 21 anos ou mais & $9(8,6 \%)$ & $21(14,2 \%)$ & $2(4,1 \%)$ & \\
\hline
\end{tabular}

Fonte: Elaboração própria

(1) Teste de Kruskall-Wallis.

(2) Valor- $p$ significativo $<0,05$.

$\mathrm{Na}$ perspectiva da enfermagem, nenhum dos três hospitais apresentou dimensões classificadas como fortes para a cultura de segurança do paciente (respostas positivas acima de 75,0\%). A equipe de enfermagem do hospital A apontou sete dimensões frágeis para a cultura de segurança do paciente (respostas positivas abaixo de 50\%). As equipes dos hospitais B e C, identificaram, cada uma, nove dimensões frágeis. As dimensões 4 - Apoio da gestão para segurança do paciente, 5 - Percepção geral da segurança do paciente, 9 - Trabalho em equipe entre as unidades, 10 - Adequação de profissionais, 11 Passagem de plantão ou de turno/transferências e 12 - Respostas não punitivas aos erros foram classificadas como áreas frágeis pela enfermagem dos três hospitais (Tabela 2).

Ao comparar a média de respostas positivas dos três hospitais, na perspectiva da equipe de enfermagem, as dimensões 4 - Apoio da gestão para segurança do paciente, 5 - Percepção geral da segurança do paciente, 6 - Retorno da informação e comunicação sobre erro, 7 - Abertura na comunicação e 11 - Passagem de plantão ou de turno/transferências apresentaram diferença estatisticamente significativa $(p<0,05)$, bem como a média global de todas as dimensões ( $p=0,013$ ) (Tabela 2). 
Tabela 2 - Comparação das respostas positivas das dimensões do Hospital Survey on Patient Safety Culture nos três hospitais sob a perspectiva da equipe da enfermagem. Divinópolis, Minas Gerais, Brasil - 2018. ( $\mathrm{N}=303)$

\begin{tabular}{|c|c|c|c|c|}
\hline \multirow[t]{2}{*}{$\begin{array}{l}\text { Dimensões do Hospital Survey on Patient } \\
\text { Safety Culture }\end{array}$} & \multicolumn{4}{|c|}{$\begin{array}{l}\text { Média \% de respostas positivas do Hospital } \\
\text { Survey on Patient Safety Culture (Desvio-padrão) }\end{array}$} \\
\hline & Hospital A & Hospital B & Hospital C & Valor $\boldsymbol{p}(1)$ \\
\hline 1 - Trabalho em equipe dentro das unidades & $59,0( \pm 32,1)$ & $48,8( \pm 32,1)$ & $53,2( \pm 34,4)$ & 0,052 \\
\hline $\begin{array}{l}2 \text { - Expectativas sobre o seu supervisor/chefe } \\
\text { e ações promotoras da segurança do paciente }\end{array}$ & $57,4( \pm 33,4)$ & $58,1( \pm 34,6)$ & $54,8( \pm 34,4)$ & 0,818 \\
\hline $\begin{array}{l}3 \text { - Aprendizado organizacional-melhoria } \\
\text { contínua }\end{array}$ & $65,2( \pm 36,5)$ & $58,3( \pm 34,6)$ & $51,0( \pm 37,9)$ & 0,059 \\
\hline $\begin{array}{l}4 \text { - Apoio da gestão para segurança do } \\
\text { paciente }\end{array}$ & $48,7( \pm 38,0)$ & $28,7( \pm 37,1)$ & $20,1( \pm 27,4)$ & $<0,001(2)$ \\
\hline 5 - Percepção geral da segurança do paciente & $42,0( \pm 28,6)$ & $31,5( \pm 25,9)$ & $35,4( \pm 25,4)$ & $0,012(2)$ \\
\hline $\begin{array}{l}6 \text { - Retorno da informação e comunicação } \\
\text { sobre erro }\end{array}$ & $54,4( \pm 36,7)$ & $43,3( \pm 35,4)$ & $27,9( \pm 30,7)$ & $<0,001(2)$ \\
\hline 7 - Abertura da comunicação & $45,7( \pm 35,3)$ & $56,4( \pm 35,7)$ & $46,3( \pm 35,2)$ & $0,037(2)$ \\
\hline 8 - Frequência de relato de eventos & $57,6( \pm 44,1)$ & $44,8( \pm 43,7)$ & $43,1( \pm 45,1)$ & 0,053 \\
\hline 9 - Trabalho em equipe entre as unidades & $37,2( \pm 32,9)$ & $34,3( \pm 31,5)$ & $34,9( \pm 25,9)$ & 0,786 \\
\hline 10 - Adequação de profissionais & $41,8( \pm 25,0)$ & $42,3( \pm 22,4)$ & $48,1( \pm 27,3)$ & 0,404 \\
\hline $\begin{array}{l}11 \text { - Passagem de plantão ou de turno/ } \\
\text { transferências }\end{array}$ & $45,2( \pm 33,9)$ & $32,5( \pm 31,1)$ & $30,1( \pm 26,2)$ & $0,004(2)$ \\
\hline 12 - Respostas não punitivas aos erros & $14,3( \pm 21,2)$ & $22,0( \pm 29,5)$ & $21,4( \pm 26,4)$ & 0,145 \\
\hline Média global & $47,4( \pm 19,8)$ & $41,7( \pm 18,8)$ & $38,4( \pm 16,9)$ & $0,013(2)$ \\
\hline
\end{tabular}

Fonte: Elaboração própria.

(1) Teste de Kruskall-Wallis.

(2) Valor-p significativo $<0,05$.

Na comparação da percepção das equipes de enfermagem dos três hospitais, por grupos dois a dois, quanto à média global, houve diferença estatística significativa entre o hospital A e B $(p=0,016)$ e entre o hospital A e C $(p=0,008)$, inferindo-se que a equipe de enfermagem do hospital A teve melhor percepção de cultura de segurança do paciente. Quanto à dimensão 4, "Apoio da gestão para segurança do paciente", houve diferença significativa dos hospitais A e B, e do hospital A e $\mathrm{C}$, ambos com $p<0,001$ (Tabela 3). Isso indica que os profissionais de enfermagem do hospital A referiram melhor apoio da gestão em comparação às outras instituições hospitalares.

Com relação à dimensão 5, "Percepção geral da segurança do paciente", observou-se diferença significativa apenas entre a percepção das equipes de enfermagem do hospital A e do hospital B $(p=0,003)$. O hospital A teve percentual de respostas positivas significativamente maior que a do hospital B $(p=0,016)$. Infere-se que os profissionais de enfermagem do hospital A apresentaram melhor percepção da segurança do paciente (Tabelas 2 e 3 ).

Ao analisar a dimensão 6, "Retorno da informação e comunicação sobre erro", houve diferença estatística significativa entre a percepção da equipe de enfermagem de todos os hospitais. O hospital C apresentou percentual de respostas positivas nesta dimensão significativamente menor em relação aos hospitais A $(p<0,001)$ e B $(p=0,007)$. Já o hospital B foi significativamente menor em comparação ao hospital A $(p=0,014)$. Infere-se que a equipe de enfermagem do hospital $\mathrm{C}$ apontou maior fragilidade na comunicação de eventos adversos. 
No que se refere à dimensão 7, "Abertura na comunicação", houve diferença estatisticamente significativa apenas entre a percepção da equipe de enfermagem do hospital A e do hospital B. O hospital B apresentou maior percentual de respostas positivas nesta dimensão em comparação a do hospital A ( $p=0,017)$ (Tabelas 2 e 3 ).

Por fim, a dimensão 11, "Passagem de plantão ou de turno/transferências", apresentou diferença estatisticamente significativa, segundo a perspectiva da equipe de enfermagem do hospital A comparado à enfermagem dos hospitais B e C (Tabela 3). Os dados mostraram que o hospital A apresentou menor fragilidade quanto à atividade de passagem de plantão ou de turno/ transferências do cuidado em comparação aos hospitais $\mathrm{B}(p=0,002)$ e $\mathrm{C}(p=0,010)$.

Tabela 3 - Comparação múltipla dos escores das dimensões 4, 5, 6, 7 e 11 e da média global do instrumento Hospital Survey on Patient Safety Culture, considerando os grupos dos hospitais dois a dois. Divinópolis, Minas Gerais, Brasil - 2018. (N=303)

\begin{tabular}{|c|c|c|c|}
\hline \multirow{2}{*}{$\begin{array}{l}\text { Dimensões do Hospital } \\
\text { Survey on Patient } \\
\text { Safety Culture }\end{array}$} & \multicolumn{3}{|c|}{ Valor- $\boldsymbol{p}$ (1) } \\
\hline & Hospital A x Hospital B & Hospital A x Hospital C & Hospital B x Hospital C \\
\hline $\begin{array}{l}4 \text { - Apoio da gestão para a } \\
\text { segurança do paciente }\end{array}$ & $<0,001(2)$ & $<0,001(2)$ & 0,250 \\
\hline $\begin{array}{l}5 \text { - Percepção geral da } \\
\text { segurança do paciente }\end{array}$ & $0,003(2)$ & 0,150 & 0,375 \\
\hline $\begin{array}{l}6 \text { - Retorno da informação } \\
\text { e comunicação sobre erro }\end{array}$ & $0,014(2)$ & $<0,001(2)$ & $0,007(2)$ \\
\hline $\begin{array}{l}7 \text { - Abertura da } \\
\text { comunicação }\end{array}$ & $0,017(2)$ & 0,917 & 0,084 \\
\hline $\begin{array}{l}11 \text { - Passagem de plantão } \\
\text { ou de turno/transferências }\end{array}$ & $0,002(2)$ & $0,010(2)$ & 0,869 \\
\hline Média Global & $0,016(2)$ & $0,008(2)$ & 0,409 \\
\hline
\end{tabular}

Fonte: Elaboração própria.

(1) Teste Mann-Whitney.

(2) Valor-p significativo considerando correção de Bonferroni $(p<0,017)$.

Identificou-se diferença estatisticamente significativa $(\mathrm{p}=0,027)$ sobre a avaliação da segurança do paciente na área/unidade de trabalho no hospital. No hospital B, a maioria dos profissionais de enfermagem $(55,6 \%)$ percebeu a segurança do paciente como regular. Com relação ao número de notificações de eventos adversos registrados nos últimos 12 meses, 65,3\% da equipe de enfermagem do hospital A, 62,2\% do hospital B e 79,6\% do hospital C relataram não ter realizado nenhuma notificação.

\section{Discussão}

As caracterizações sociodemográfica e profissional dos participantes deste estudo convergem com achados similares de outros estudos brasileiros na temática, que identificaram predominância do sexo feminino entre os profissionais de enfermagem, a maioria pertencente à categoria profissional de técnicos ou auxiliares de enfermagem, com ensino médio completo, carga horária de trabalho elevada, maior que 40 horas semanais, tempo menor que cinco anos de atuação na instituição e vínculo empregatício exclusivo $^{(7,15-16)}$. O recurso humano e suas características são considerados fatores que podem influenciar na assistência segura ${ }^{(7)}$.

$\mathrm{Na}$ perspectiva da equipe de enfermagem, nenhum dos três hospitais em estudo apresentou dimensões positivas para a cultura de segurança do paciente do HSOPSC da AHRQ ${ }^{(4)}$. Pesquisas brasileiras desenvolvidas com equipes de enfermagem também identificaram uma cultura frágil de segurança do paciente $(49,2 \%)^{(7)}$ e $(45 \%)^{(16)}$. Estudos internacionais, sendo um conduzido na Hungria e outro na Malásia, encontraram uma cultura frágil de segurança do paciente, $52,2 \%{ }^{(17)}$ 
e $50,1 \%{ }^{(18)}$, respectivamente. Nota-se, portanto, que os desafios relacionados à melhoria da cultura de segurança do paciente em instituições hospitalares ocorrem regional, nacional e internacionalmente.

Ao considerar as dimensões do HSOPSC que se apresentaram frágeis sob a percepção da equipe de enfermagem nos três hospitais estudados, destaca-se a dimensão 4, "Apoio da gestão para segurança do paciente". A liderança organizacional tem papel fundamental na construção e consolidação de uma cultura na qual os erros e as falhas são utilizados como forma de conhecimento e de aprendizagem contínua ${ }^{(19)}$. Do mesmo modo, a liderança de enfermagem é fator crítico para o sucesso de uma assistência de enfermagem segura.

Estudos brasileiros desenvolvidos com equipes de enfermagem também identificaram fragilidade na dimensão 5, "Percepção geral da segurança do paciente" ${ }^{(7,16)}$. Observa-se similaridade em outros dois estudos envolvendo equipe multiprofissional, sendo que um dos estudos foi conduzido em hospitais com gestões diferentes $^{(20-21)}$. Esse achado pode estar relacionado à vulnerabilidade na realização de procedimentos e de processos de mitigação de riscos e erros, o que aponta fragilidades em unidades de internação na área hospitalar que podem impactar na segurança do paciente.

A dimensão 9, "Trabalho em equipe entre as unidades", também foi considerada uma área de fragilidade para a cultura de segurança do paciente pelas equipes de enfermagem dos três hospitais. Esse resultado remete a uma dificuldade de cooperação e comunicação entre os setores, necessários para o bom andamento do trabalho. Desenvolver um clima de trabalho harmonioso, de respeito e cooperativo, é importante, pois os profissionais sentem-se valorizados e respeitados, colaborando no trabalho em equipe e na prestação de um cuidado de qualidade aos pacientes $^{(19)}$.

A dimensão 10, "Adequação de profissionais", também apontada como frágil pela equipe de enfermagem, é influenciada pelo quantitativo de profissionais atuantes nas instituições de saúde.
O dimensionamento inadequado de profissionais influencia negativamente na avaliação da segurança do paciente. Estudo realizado na Inglaterra evidenciou que a redução quantitativa da equipe de enfermagem esteve associada a maior risco de morte durante uma internação hospitalar. O risco de morte foi aumentado em 3\% para cada dia em que se teve um dimensionamento da equipe de enfermagem abaixo do necessário ${ }^{(22)}$.

Outro aspecto importante para a cultura de segurança do paciente é abordado na dimensão 11, "Passagem de plantão ou de turno/transferências", que, neste estudo, apresentou-se fragilizada na percepção dos profissionais de enfermagem dos três hospitais. A passagem de plantão ou turno pode ser comprometida por diversos fatores, como aumento de carga de trabalho, omissão de dados dos pacientes, interrupções e conversas paralelas envolvendo outras pessoas durante a execução de uma atividade principal ${ }^{(18)}$. Achados similares apontam que a passagem de plantão realizada pela enfermagem pode assegurar a continuidade do cuidado e garantir a segurança do paciente, pois permite a transmissão da evolução clínica para continuidade dos cuidados específicos. Para tanto, é necessária a padronização da transmissão das informações, por meio de protocolos e ferramentas ${ }^{(3,7,20)}$.

A dimensão 12, "Respostas não punitiva aos erros", também foi apontada como fragilidade na cultura de segurança pela equipe de enfermagem dos três hospitais. Este resultado corrobora investigações de outros estudos que evidenciaram a necessidade de mudança da cultura da culpabilidade para um processo baseado no aprendizado com os erros dentro das organizações $^{(5,15-18)}$. Faz-se necessário, portanto, o estímulo ao processo de notificação dos eventos adversos nas instituições, a fim de favorecer uma comunicação mais efetiva e, principalmente, propiciar o aprendizado constante dos profissionais envolvidos ${ }^{(7,23)}$.

No que concerne à comparação das 12 dimensões do instrumento HSOPSC, sob a percepção das equipes de enfermagem dos três hospitais, destaca-se o hospital filantrópico (hospital A), por ter apresentado melhor percepção da cultura 
de segurança do paciente em quatro dimensões. A referida instituição também apresentou melhor média global de cultura de segurança do paciente em comparação às demais instituições (B e C), as quais possuem gestão pública. Tal diferença pode estar relacionada ao ambiente de trabalho hospitalar, a exemplo de condições físicas inadequadas, característica encontrada, principalmente, em hospitais públicos. Em contrapartida, as instituições privadas, assim como as de natureza filantrópica, visam o alcance de metas econômicas e financeiras e, consequentemente, investem em estratégias que reduzam custos advindos de falhas e erros relacionados às prestações de serviços em saúde ${ }^{(21)}$. Portanto, hospitais privados tendem a apresentar melhor avaliação para a cultura de segurança do paciente em relação a hospitais de gestão estadual ou federal ${ }^{(21)}$.

A maioria dos profissionais de enfermagem deste estudo evidenciou uma percepção geral de cultura de segurança do paciente classificada como regular. Este resultado difere dos achados de pesquisa desenvolvida com enfermeiros na Turquia ${ }^{(19)}$, que identificou uma percepção geral de cultura de segurança do paciente positiva $(50,2 \%)$, considerada como muito boa ou excelente. Contudo, a percepção de cultura de segurança negativa encontrada no presente estudo assemelha-se com outra pesquisa brasileira que apontou que $47,9 \%$ dos profissionais avaliaram como regular, $10,3 \%$ ruim e $12,0 \%$ muito ruim ${ }^{(21)}$. Para a mudança desse cenário, são necessárias estratégias de melhorias nos processos de trabalho dos profissionais, envolvendo o gerenciamento de risco, dimensionamento adequado de número de profissionais, treinamentos da equipe acerca da segurança do paciente, melhoria no sistema de notificação alicerçada na comunicação aberta e garantia de aprendizado organizacional contínuo $^{(5,7)}$.

O estudo revelou baixa notificação de eventos adversos nos últimos 12 meses pelos profissionais de enfermagem nos três hospitais, o que remete também à dimensão 6, "Retorno da informação e comunicação sobre erro", apontada como fragilizada. Isso demonstra que ainda não foi estabelecida uma comunicação aberta sobre a ocorrência de eventos adversos relacionados à assistência à saúde. Portanto, há uma subnotificação dos eventos adversos nos hospitais, o que pode impactar de forma negativa na qualidade da assistência prestada ao paciente. Os fatores associados à subnotificação são: sobrecarga de trabalho, falta de conhecimento do sistema de notificação, medo devido à cultura punitiva ao erro, diferenças organizacionais e culturais e falta de políticas que incentivem a comunicação do erro ${ }^{(7,17,19-20)}$.

Como limitação do estudo, tem-se a obtenção da avaliação da cultura de segurança do paciente apenas pela perspectiva da equipe de enfermagem, visto que a cultura de segurança abrange toda a equipe multiprofissional atuante no cenário hospitalar. Além disso, as conclusões não devem ser generalizados, visto que o estudo foi realizado em três hospitais de referência regional com características distintas. A singularidade de uma categoria profissional e as diferentes realidades institucionais podem impactar diretamente na percepção sobre a cultura de segurança do paciente. Assim, são necessários outros estudos com avaliações multiprofissionais, em que se considerem os diferentes contextos institucionais.

Em contrapartida, os achados deste estudo contribuirão para o direcionamento de intervenções específicas para as áreas consideradas frágeis pela equipe de enfermagem em cada instituição cenário do estudo. O fortalecimento da percepção da cultura de segurança do paciente entre os membros da equipe de enfermagem, que constitui o maior grupo de profissionais das instituições hospitalares, poderá impactar na melhoria de cultura de segurança do paciente de forma geral.

\section{Conclusão}

As equipes de enfermagem dos três hospitais, cenário deste estudo, revelaram fragilidades em todas as dimensões do instrumento HSOPSC sobre a cultura de segurança do paciente. É necessário que os gestores e a liderança de enfermagem desenvolvam estratégias para o fortalecimento da 
cultura de segurança nos hospitais com foco na melhoria contínua da assistência.

A maioria dos profissionais de enfermagem dos hospitais analisados classificaram a segurança do paciente como regular, porém a cultura de segurança do paciente mostrou-se mais fortalecida no hospital A, filantrópico. Isso sugere possível influência da filosofia e da gestão dos serviços na percepção sobre a cultura de segurança.

A dimensão sobre comunicação do erro foi frágil nos três hospitais, reforçada pelo fato de que, nos últimos 12 meses, poucos profissionais de enfermagem fizeram algum relato de evento adverso, o que evidencia a necessidade de quebra de paradigma de uma cultura punitiva, ainda prevalecente, para uma cultura pautada no aprendizado com base nos erros.

Os achados do estudo trazem importantes contribuições para os gestores hospitalares e para a ciência da enfermagem, pois aponta fragilidades nos hospitais e pode ser instrumento para melhorias no fortalecimento da cultura de segurança. Recomenda-se a reprodutibilidade de pesquisas semelhantes em diferentes serviços de saúde e a inclusão das demais categorias profissionais na avaliação, para inspirar a liderança hospitalar sobre esta temática.

\section{Colaborações:}

1 - concepção, projeto, análise e interpretação dos dados: Graziele de Carvalho Lemos, Luciana Regina Ferreira da Mata, Helen Cristiny Teodoro Couto Ribeiro e Aline Carrilho Menezes;

2 - redação do artigo e revisão crítica relevante do conteúdo intelectual: Graziele de Carvalho Lemos, Luciana Regina Ferreira da Mata, Helen Cristiny Teodoro Couto Ribeiro, Aline Carrilho Menezes, Carolina de Sousa Penha, Ronise Malaquias Carlos Valadares e Marlene Santos Rios Castro;

3 - aprovação final da versão a ser publicada: Graziele de Carvalho Lemos, Luciana Regina Ferreira da Mata, Helen Cristiny Teodoro Couto Ribeiro, Aline Carrilho Menezes, Carolina de Sousa Penha, Ronise Malaquias Carlos Valadares e Marlene Santos Rios Castro.

\section{Referências}

1. World Health Organization. Global Patient Safety Action Plan 2021-2030: Towards eliminating avoidable harm in health care [Internet]. Geneva (CH); 2021 [cited 2022 Jan 5]. Available from: https://www.who.int/teams/ integrated-health-services/patient-safety/policy/ global-patient-safety-action-plan.

2. Ramírez-Martínez ME, Pedraza-Avilés AG. Cultura de seguridad y eventos adversos en una clínica de primer nivel. Enferm univ. 2017;14(2):111-7. DOI: https://doi.org/10.1016/j.reu.2017.02.006

3. Reis CT, Paiva SG, Sousa P. The patient safety culture: a systematic review by characteristics of Hospital Survey on Patient Safety Culture dimensions. Int J Qual Health Care. 2018;30(9): 660-77. DOI: https://doi.org/10.1093/intqhc/mzy 080

4. Sorra JS, Nieva VF. Hospital Survey on Patient Safety Culture (Prepared by Westat, under Contract N. 290-96-0004). AHRQ Publication No. 04-0041 [Internet]. Rockville (MD): Agency for Healthcare Research and Quality; 2004 [cited 2021 Feb 11]. Available from: https://proqualis.net/sites/ proqualis.net/files/User\%20guide\%20HSOPSC.pdf

5. Aouicha W, Tlili MA, Sahli J, Dhiab MB, Chelbi S, Mtiraoui A, et al. Exploring patient safety culture in emergency departments: A Tunisian perspective. Int Emerg Nurs. 2021;54:100941. DOI: 10.1016/ j.ienj.2020.100941

6. Butler GA, Hupp DS. Pediatric Quality and Safety: A Nursing Perspective. Pediatr Clin North Am. 2016;63(2):329-39. DOI: 10.1016/j.pcl.2015.11.005

7. Sanchis DZ, Haddad MCFL, Girotto E, Silva AMR. Cultura de segurança do paciente: percepção de profissionais de enfermagem em instituições de alta complexidade. Rev Bras Enferm. 2020;73(5):e20190174. DOI: 10.1590/0034-71672019-0174

8. Flin R. Measuring safety culture in healthcare: A case for accurate diagnosis. Saf Sci 2007;45(6): 653-67. DOI: 10.1016/j.ssci.2007.04.003

9. Agency for Healthcare Research and Quality. Surveys on Patient Safety Culture Research Reference List. Content last reviewed April 2017 [Internet]. Rockville (MD): AHRQ; 2017 [cited 2021 Feb 11]. Available from: http://www. ahrq.gov/professionals/quality-patient-safety/ patientsafetyculture/resources/index.html 
10. Reis CT, Laguardia J, Martins M. Adaptação transcultural da versão brasileira do Hospital Survey on Patient Safety Culture: etapa inicial. Cad Saúde Pública. 2013;28(11):2199-210. DOI: https:// doi.org/10.1590/S0102-311X2012001100019

11. Reis CT, Laguardia J, Vasconcelos AGG, Martins M. Reliability and validity of the Brazilian version of the Hospital Survey on Patient Safety Culture (HSOPSC): a pilot study. Cad Saúde Pública. 2016;32(11):e00115614. DOI: https://doi.org/10.1590/ 0102-311x00115614

12. Von Elm E, Altman DG, Egger M, Pocock SJ, Gøtzche PC, Vandenbroucke JP. The Strengthening the Reporting of Observational Studies in Epidemiology (STROBE) statement: Guidelines for Reporting Observational Studies. PLoS Med. 2007;4(10):e296. DOI: https://doi.org/10.1371/journal. pmed.0040296

13. Hulley SB, Cummings SR, Browner WS. Designing clinical research: an epidemiologic approach. 2th ed. Philadelphia: Lippincott Williams \& Wilkins; 2001.

14. Fonseca JS, Martins GA. Curso de estatística. $5 \mathrm{a}$ ed. São Paulo: Atlas; 1994.

15. Melo E, Balsanelli AP, Neves VR, Bohomol E. Cultura de segurança do paciente segundo profissionais de enfermagem de um hospital acreditado. Rev Gaúcha Enferm. 2020;41:e20190288. DOI: https://doi.org/10.1590/1983-1447.2020.2019 0288

16. Macedo TR, Rocha PK, Tomazoni A, Souza S, Anders JC, Davis K. Cultura de segurança do paciente na perspectiva da equipe de enfermagem de emergências pediátricas. Rev esc enferm USP. 2016;50(5):756-62. DOI: https://doi.org/10.1590/ s0080-623420160000600007

17. Granel N, Manresa-Dominguez JM, Barth A, Papp K, Bernabeu-Tamayo MD. Patient safety culture in Hungarian hospitals. Int $\mathrm{J}$ Health Care
Qual Assur. 2019;11;32(2);412-24. DOI: $10.1108 /$ IJHCQA-02-2018-0048

18. Jye AKR, Hing CZ, Peter S, Bartholomew P, Senok J. Hospital survey on patient safety culture in Sarawak General Hospital: A cross sectional study. Med J Malaysia [Internet]. 2019 [cited 2021 Feb 11];74(5):385-8. Available from: http://www. e-mjm.org/2019/v74n5/patient-safety-culture.pdf

19. Günes ÜY, Gürlek Ö, Sönmez M. A survey of the patient safety culture of hospital nurses in Turkey. Collegian. 2016;23(2):225-32. DOI: 10.1016/j.colegn. 2015.02.005

20. Minuzzi AP, Salum NC, Locks MOH. Avaliação da cultura de segurança do paciente em terapia intensiva na perspectiva da equipe de saúde. Texto contexto - enferm. 2016;25(2):e1610015. DOI: https://doi.org/10.1590/0104-0707201600161 0015

21. Andrade LEL, Lopes JM, Souza Filho MCM, Vieira Junior RF, Farias LPC, Santos CCM, et al. Cultura de segurança do paciente em três hospitais brasileiros com diferentes tipos de gestão. Ciênc saúde colet. 2018;23(1):161-72. DOI: https://doi.org/10.1590/1413-81232018231. 24392015

22. Griffiths P, Maruotti A, Saucedo AR, Redfern OC, Ball JE, Briggs J, et al. Nurse staffing, nursing assistants and hospital mortality: retrospective longitudinal cohort study. BMJ Qual Saf. 2018;28(8):609-17. DOI: 10.1136/bmjqs-2018-008 043

23. Notaro KAM, Corrêa AR, Tomazoni A, Rocha PK, Manzo BF. Cultura de segurança da equipe multiprofissional em Unidades de Terapia Intensiva Neonatal de hospitais públicos. Rev Latino-Am Enfermagem. 2019;27:e3167. DOI: https://doi. org/10.1590/1518-8345.2849.3167

Recebido: 16 de junho de 2021

Aprovado: 9 de dezembro de 2021

Publicado: 16 de fevereiro de 2022

A Revista Baiana de Enfermagem utiliza a Licença Creative Commons - Atribuição-NãoComercial 4.0 Internacional. https://creativecommons.org/licenses/by-nc/4.0/ Este artigo é de acesso aberto distribuído sob os termos da Licença Creative Commons (CC BY-NC). Esta licença permite que outros remixem, adaptem e criem a partir do seu trabalho para fins não comerciais. Embora os novos trabalhos tenham de lhe atribuir o devido crédito e não possam ser usados para fins comerciais, os usuários não têm de licenciar esses trabalhos derivados sob os mesmos termos. 